\title{
AIDS-Related Kaposi Sarcoma I (Immune System) Status
}

National Cancer Institute

\section{Source}

National Cancer Institute. AIDS-Related Kaposi Sarcoma I (Immune System) Status. NCI

Thesaurus. Code C134978.

A term that refers to the immune system status of AIDS-related Kaposi sarcoma according to the AIDS Clinical Trials Group system. The immune status is assessed using the $\mathrm{CD} 4$ count test. (American Cancer Society) 